\title{
Michael Jones (éd.), Le premier inventaire du Trésor des chartes des ducs de Bretagne (1395)
}

Société d'histoire et d'archéologie de Bretagne, Rennes, 2007, 320 p.

\section{Yves Coativy}

\section{(2) OpenEdition}

\section{Journals}

\section{Édition électronique}

URL : http://journals.openedition.org/abpo/126

DOI : $10.4000 / a b p o .126$

ISBN : 978-2-7535-1514-7

ISSN : 2108-6443

Éditeur

Presses universitaires de Rennes

\section{Édition imprimée}

Date de publication : 30 juin 2009

Pagination : 233-235

ISBN : 978-2-7535-0941-2

ISSN : 0399-0826

\section{Référence électronique}

Yves Coativy, « Michael Jones (éd.), Le premier inventaire du Trésor des chartes des ducs de Bretagne (1395) ", Annales de Bretagne et des Pays de l'Ouest [En ligne], 116-2 | 2009, mis en ligne le 31 décembre 2009, consulté le 20 septembre 2020. URL : http://journals.openedition.org/abpo/126 ; DOI : https:// doi.org/10.4000/abpo.126

Ce document a été généré automatiquement le 20 septembre 2020.

(C) Presses universitaires de Rennes 


\section{Michael Jones (éd.), Le premier inventaire du Trésor des chartes des ducs de Bretagne (1395)}

Société d'histoire et d'archéologie de Bretagne, Rennes, 2007, 320 p.

\section{Yves Coativy}

\section{RÉFÉRENCE}

Société d'histoire et d'archéologie de Bretagne, Rennes, 320 p.

Après les actes de la duchesse Constance (en collaboration avec Judith Everard), de Charles de Blois et de Jean IV, Michael Jones poursuit la publication de sources essentielles de l'histoire de la Bretagne. Il livre cette fois-ci au public l'inventaire des archives ducales dressé en 1395 par Hervé Le Grant. Le manuscrit original est conservé aux Archives départementales de Loire-Atlantique, une première transcription en a été faite à Brest en 1998 par Claire Lagadec dans le cadre d'un mémoire de maîtrise sous la direction de Jean Kerhervé. Il mentionne environ 900 documents concernant la Bretagne ducale des XIII ${ }^{e}$ et $\mathrm{XIV}^{\mathrm{e}}$ siècles. Si l'historiographie de la Bretagne naît au XIV siècle avec les ducs de la maison de Montfort, on peut situer plus précocement la " révolution documentaire » bretonne dans le courant du XIII ${ }^{\mathrm{e}}$ siècle, sous le règne de Jean le Roux (1237-1286). C'est en effet alors que les pouvoirs se mettent à utiliser abondamment l'écrit pour conserver la mémoire de leurs actes, qu'apparaissent les premiers notaires, que les méthodes de datation changent, etc. Si nous percevons les marques de ce phénomène, nous ne pouvons que constater le vide documentaire pour cette époque, dû en grande partie à la guerre de Succession (1341-1364) qui a des effets désastreux sur la conservation des archives, rappelons par exemple la destruction en 1363 des archives conservées lors du sac et de l'incendie de la forteresse ducale de Suscinio (56), d'où l'intérêt fondamental des inventaires anciens. 
2 L'ouvrage commence par une longue introduction en deux parties. La première consiste en la présentation du document, des inventaires postérieurs et de l'auteur, Hervé Le Grant. L'inventaire de 1395 apparaît comme la mémoire officielle de l'État breton, Dreux et Montfort, à un moment bien particulier, le milieu des années 1390. À cette époque, le duc est en conflit larvé avec le roi de France et cherche à prouver par enquêtes et débats sa souveraineté sur la Bretagne. Ce travail se fait d'ailleurs en parallèle avec les débuts de l'historiographie bretonne : Jean IV et son administration usent de tous les moyens à leur disposition pour essayer d'enrayer l'expansionnisme royal dans le duché.

3 L'habitude est rapidement prise d'inventorier et de classer les actes du pouvoir devient rapidement une habitude en Bretagne. $\mathrm{M}$. Jones pointe ainsi des recensions faites en 1430, dans les années 1450, en 1464, et encore dans les années 1470. À chaque fois bien évidemment, les corpus sont complétés par les documents entrés au Trésor depuis le travail précédent. La chronologie est tout à fait révélatrice car il s'agit presque systématiquement de périodes durant lesquelles le pouvoir ducal est en lutte plus ou moins ouverte avec le roi de France, ce qui confirme tout ce que Michael Jones écrit sur le rôle éminemment politique de la conservation et du classement des archives. Il existe par ailleurs de petits inventaires thématiques qui permettent aux juristes bretons de défendre tel ou tel aspect des droits du duc. D'ailleurs, dès son arrivée au pouvoir en 1488, la duchesse Anne demande "d'inventoriser» (sic) le Trésor des chartes. Les événements ne le permettent sans doute pas et la liste suivante date du début $\mathrm{du} \mathrm{xvI}^{\mathrm{e}}$ siècle. Le travail est repris de multiples fois sous l'Ancien Régime mais les motivations ont changé : il s'agit désormais plus de conserver une mémoire que de défendre les droits de la Bretagne, encore que cet aspect des choses joue sans doute un rôle, concernant les privilèges de la province. C'est en 1870 que l'archiviste Léon Maître abandonne le système de classement hérité du Moyen Âge pour un nouveau classement et, de fait, un nouvel inventaire, bien conforme aux standards contemporains. Les premières liasses de la série $\mathrm{E}$ des Archives départementales de Loire-Inférieure regroupent les 4090 documents du Trésor qui ont survécu. À cette occasion, certaines pièces sont distraites de l'ensemble (souvent des doubles) pour être expédiées vers les autres centres d'archives bretons. Le dernier épisode de cette aventure date de 1969 : cette année-là, Léon Rouzeau réalise un recollement et un estampillage complet du Trésor, avec une nouvelle numérotation des pièces, toujours valable aujourd'hui.

M. Jones compare le cas breton avec celui des autres pouvoirs, royaux et princiers, de l'Occident médiéval. C'est après la défaite de Philippe Auguste à Fréteval (1194) et la perte d'une partie de ses bagages, dont ses chartes, qu'il devient évident qu'il faut conserver correctement les archives royales. Le Dauphin de Viennois fait quant à lui inventorier ses parchemins en 1277. C'est à la même époque que la Bourgogne commence à se préoccuper des siennes, avec des inventaires en 1280 et 1304 . La Bretagne est plus lente à recencer ses archives puisqu'il faut attendre la fin du XIV siècle pour trouver notre inventaire, mais il faut reconnaître que nous sommes mal renseignés sur la situation antérieure à la guerre de Succession.

L'étude se prolonge, comme il se doit, d'une présentation de la diplomatique des actes, du mode de scellement, des modes de conservation, etc. puis Michal Jones rappelle ce que l'on sait de celui qui a effectué le travail. Hervé Le Grant naît vers 1360 et arrive à Nantes vers 1373, où il apprend d'un oncle son métier de notaire. À la fin des années 1370, il entre au service de Jean IV et il travaille avec Guillaume de Saint-André, le 
biographe de Jean IV. Ses occupations ne se limitent pas à la rédaction d'actes pour le duc. Il est aussi amené à réfléchir aux droits de son souverain tout en vidimant, c'est-àdire en recopiant, des actes anciens qui peuvent appuyer sa démarche. Vers 1385, il devient secrétaire ducal et assiste à de nombreuses négociations diplomatiques. Ses services sont appréciés puisqu'il est nommé à la tête d'une ambassade qui se rend auprès du roi d'Angleterre (mai 1384). En 1395, il reçoit la garde des archives ducales, qu'il inventorie donc, et où il fait régner une ambiance laborieuse. Le début du $\mathrm{XV}^{\mathrm{e}}$ siècle se caractérise en effet par une grande dépense d'énergie au Trésor des chartes. Les documents sont classés, recopiés, analysés, etc. Quand Hervé Le Grant estime qu'il manque des documents importants, il les fait rechercher et copier pour compléter le fonds dont il a la garde. Dans le même temps, il rédige un important formulaire, une liste d'actes type pouvant servir en telle occasion à défendre les droits du duc. Il est toujours en activité le 3 janvier 1416 mais meurt sans doute cette année-là car il est remplacé huit mois plus tard.

6 Dans la seconde partie de son introduction, Michael Jones met ces éléments en relation avec ce que l'on sait de l'auteur de la Chronique de Saint-Brieuc, en partie d'après les travaux de Jean Kerhervé. Fervent patriote breton, au service de Jean IV dont il approuve globalement la politique, c'est un homme d'Eglise mais aussi un bon serviteur de l'État, bien au fait des pratiques financières et juridiques de la Bretagne de la fin du $\mathrm{XIV}^{\mathrm{e}}$ siècle. Il rédige son ouvrage dans les années 1394-1416 mais il n'a pas le temps de le terminer. Au terme d'une démarche tout à fait convaincante, $\mathrm{M}$. Jones n'hésite pas à rapprocher l'auteur de l'inventaire et l'auteur de la Chronique. En effet, de nombreux éléments laissent à penser qu'Hervé Le Grant est l'Anonyme : il est au service de l'État breton des années 1380 aux années 1410 ; il travaille à plein-temps à la chancellerie ducale ; il est en charge du Trésor des chartes pendant les vingt dernières années de sa vie. La comparaison de ce que l'on sait sur la carrière d'Hervé Le Grant et des éléments biographiques de l'auteur de la Chronique montre d'étranges similitudes. Michael Jones emporte l'adhésion du lecteur en décorticant trois faux actes rapportés par l'Anonyme et ayant pour but de prouver les droits souverains du duc de Bretagne, à savoir une lettre d'Alain, roi de Bretagne, 689, une autre d'Alain Fergent, années 1080, et le faux traité d'Angers entre Pierre de Dreux et Louis IX, 1231. Il montre bien à quel point la parfaite connaissance des archives d'Hervé Le Grant en fait très certainement l'auteur.

7 Vient ensuite la transcription de l'inventaire. Chaque document mentionné dans l'inventaire est repéré quand il a survécu en original ou sous forme de copie, soit environ la moitié des occurrences. L'essentiel est conservé à Nantes dans le Trésor des chartes des ducs de Bretagne aux Archives départementales de Loire-Atlantique (série E). En plus des notes érudites, l'ouvrage comprend une bibliographie et un index. L'inventaire à proprement parler est organisé par grands chapeaux : propriétés des ducs de Bretagne, relations avec la noblesse bretonne (Rohan, Rays, etc.), avec les évêques et avec les rois de France et d'Angleterre, documents financiers et événements particuliers (régale de l'évêché de Quimper dans les années 1380 ; relations entre le duc, l'évêque et les habitants de Saint-Malo), sans compter de nombreux actes touchant des sujets variés, regroupés par qualité (actes scellés sur lacs de soie par exemple) ou « en vrac ».

8 L'auteur nous pardonnera quelques critiques vénielles. Il a suivi l'usage anglais qui veut que la table des matières soit située au début du livre. Quitte à y placer un sommaire, il aurait sans doute mieux valu respecter les habitudes françaises en la plaçant en fin 
d'ouvrage. Les siècles sont numérotés en chiffres arabes. Là encore, respectons la tradition et utilisons des chiffres latins. Enfin, la numérotation des notes aurait été plus claire si elle avait été la même que celle des paragraphes de l'inventaire, d'autant que le décalage n'est pas très important. Mais ce sont là des remarques de forme. Comme à son habitude, Michael Jones nous livre un ouvrage fondamental sur la Bretagne de la fin du Moyen Âge, qui va bien au-delà de son titre. En effet, non content de publier l'inventaire d'Hervé Le Grant, il le complète d'un impressionnant appareil critique et d'une introduction qui est un modèle du genre. Espérons simplement que la dédicace du livre, «In memoriam Jean IV, duc de Bretagne 1364-1399 et aux archivistes de son Trésor des chartes ", en l'honneur de l'homme sur lequel il a travaillé une grande partie de sa vie, n'annonce pas la fin de ses travaux si érudits et si utiles. 